\title{
The use of the method of controlling the electrochemical parameters of aqueous solutions to combat complications in the operation of oil field pipelines
}

\author{
Oleg Latypov ${ }^{1, *}$, Sergey Cherepashkin ${ }^{1}$, and Dina Latypova ${ }^{1}$ \\ ${ }^{1}$ Ufa State Petroleum Technological University, the Chair «Material science and protection against corrosion», Ufa, Russia
}

\begin{abstract}
Corrosion of equipment in the oil and gas complex is a global problem, as it contributes to huge material costs and global disasters that violate the environment. Corrosion control methods used to protect equipment do not always ensure the absolute safety of the operation of oil and gas facilities. Moreover, they are quite expensive. The developed method for controlling the electrochemical parameters of aqueous solutions to combat complications during the operation of oil-field pipelines provides the necessary protection against corrosion. The method is economical and environmentally friendly, since it does not require the use of chemical reagents. The test results have shown a very high efficiency in dealing with complications in oil fields.
\end{abstract}

\section{Introduction}

The most significant factor contributing to the destruction of oil and gas pipeline materials is the high corrosiveness of the production environment caused by the presence of inorganic salts, mechanical impurities, inclusions of organic substances, as well as corrosive aerobic and anaerobic microorganisms [1]. The fight against complications in the operation of oil field pipelines usually comes down to the use of chemicals (corrosion and scale inhibitors, biocides) that are pumped into technological environments. However, their presence in technological media is undesirable, since it leads to the need for further purification of oil and oil products from them.

Currently, due to the intensification of oil production, it is characterized by a high degree of water cut. As the practice of oilfield equipment operation shows, the presence of water in process fluids promotes the active development of corrosion [2]. High aggressiveness of process fluids is associated with the presence of dissolved gases, mechanical impurities, salt ions and microorganisms in them. The main cause of salt deposition on the inner surface of the equipment is the high content of active $\mathrm{Ca}^{2+}$ ions. Thus, the presence of $\mathrm{Ca}^{2+}$ ions in the process fluid in the presence of sulfates contributes to the formation of hard-to-dissolve gypsum deposits on the surface of oilfield equipment [3]. The use of fresh and waste water pumped into productive formations using reservoir pressure maintenance systems leads to their contamination with various microorganisms. It has been established that microorganisms can participate both directly in the anodic corrosion process, destroying the metal with the formation of corrosion pits, and in the cathodic depolarization process, when the products of their metabolism (for example, hydrogen sulfide) or the microorganisms themselves act as a depolarizer [4-5].

Protection methods aimed at reducing the rate of corrosion and scale deposition, suppressing the vital activity of microorganisms and separating an oil-water emulsion can be classified into reagent and non-reagent. The reagent methods include the use of various chemical compounds (corrosion and scale inhibitors, biocides, demulsifiers, etc.). Reagent-free methods include methods of influencing the production environment with magnetic and acoustic fields.

In recent years, reagent-free methods of dealing with complications in the processes of oil extraction and preparation have been developed, which are based on the use of energy-efficient technologies that contribute to a positive change in the properties of technological media. Such methods include ultrasonic action on oil fluids, lowering their temperature and pressure, the use of magnetohydrodynamic treatment (MHD) and a number of others. However, the use of many reagent-free methods in oil fields is very limited, which is associated with an insufficient study of the theoretical base and calculation methods for creating the corresponding units and devices, as well as the lack of stable and effective results of their application.

We developed a method for controlling the electrochemical parameters of field media to reduce their aggressive effect on the metal of pipelines. To implement the method, special units have been developed that allow, by changing the values of the hydrogen index and the redox potential in the volume of the process fluid stream and at the inner surface of the metal equipment, to control the mechanism and kinetics of the processes responsible for the occurrence and manifestation of the main types of complications. At the same time, the flow of the water-salt solution in the unit

\footnotetext{
* Corresponding author: o.r.latypov@,mail.ru
} 
is divided into catholyte (water-salt solution saturated with hydroxyl ions) and anolyte (water-salt solution saturated with hydroxonium ions), the introduction of a certain amount of which into the process fluid allows one to purposefully change its electrochemical characteristics and ionic composition.

\section{Research and results}

It is known that some ions have anomalously high speeds of movement, and, therefore, abnormally high mobility, especially in the presence of an electric field. These ions include hydronium ions $\mathrm{H}_{3} \mathrm{O}^{+}$and hydroxyl ions $\mathrm{OH}^{-}$. The flow of the medium in the electric field is divided into two oppositely charged flows - anolyte and catholyte. Positively charged ions prevail in anolyte, negatively charged ones in catholyte [6-7].

We have investigated the dependence of the change in the electrochemical parameters of liquids on the current strength and the duration of its exposure. To study this dependence, a special laboratory bench was developed (Fig. 1).

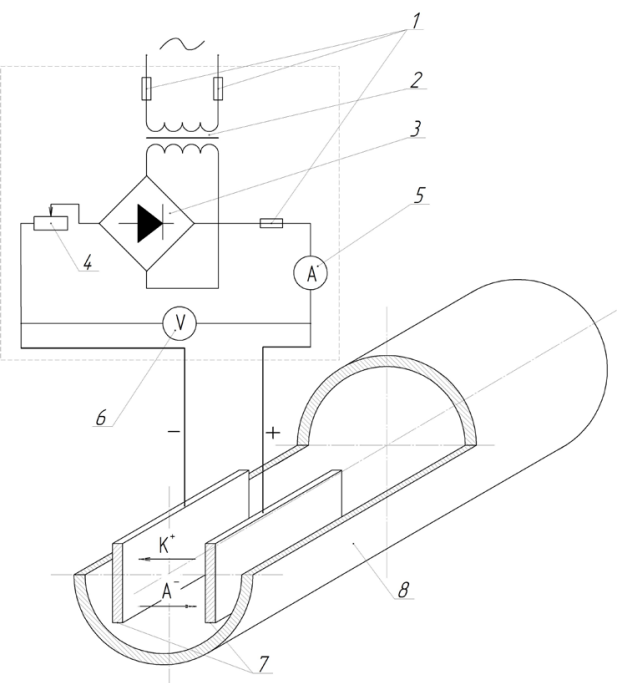

1 - fuses; 2 - step-down transformer; 3 - semiconductor rectifier; 4 - rheostat; 5 - ammeter; 6 - voltmeter; 7 - steel electrodes; 8 - pipeline with electrolyte solution

Fig 1. Laboratory stand for changing $\mathrm{pH}$ and $\mathrm{Eh}$ of water-salt solution.

A current of $24 \mathrm{~V}$ was passed through the cell electrodes for $30 \mathrm{~s}$. Samples of anolyte and catholyte were taken near the electrodes and the $\mathrm{pH}$ and Eh values were measured. The relative error in measuring $\mathrm{pH}$ and Eh was no more than $0.5 \%$.

The main factors affecting the $\mathrm{pH}$ and Eh values of anolyte and catholyte are the current strength at the electrodes and the duration of electrification of the water-salt solution. As a result of statistical processing of the experimental data, it was found that the greatest influence of the current strength on the change in the $\mathrm{pH}$ and Eh of the anolyte and catholyte appears at its value of about 2 A. slower. A high current density appears on the electrodes, which promotes the process of overvoltage and polarization of the electrode surface. As a result of polarization, charge transfer through the "metal - electrolyte" boundary layer becomes more difficult, which leads to an increase in energy consumption, contributes to the heating of the electrodes and an increase in their corrosion rate.

To study the effect of the duration of electrification of the water-salt solution on the $\mathrm{pH}$ and $\mathrm{Eh}$ of the anolyte and catholyte, a direct current of 2 A was applied to the cell electrodes. At certain time intervals, samples of the anolyte and catholyte were taken from the cell and the $\mathrm{pH}$ and Eh were measured. As a result of statistical processing of experimental data, it was found that the greatest change in $\mathrm{pH}$ and Eh occurs in the initial period of electrification of the water-salt solution for $20 \ldots 30 \mathrm{~s}$. This is due to the abnormally high mobility of hydroxyl ions and hydronium ions.

Over time, the $\mathrm{pH}$ and $\mathrm{Eh}$ of the anolyte and catholyte return to their original values. In this regard, studies were carried out on the time of the stable state of $\mathrm{pH}$ and $\mathrm{Eh}$ of anolyte and catholyte. It was found that the anolyte in air maintains the stability of $\mathrm{pH}$ and $\mathrm{Eh}$ for 30 $\mathrm{min}$, and the catholyte for $1 \mathrm{~h}$. If the contact of the catholyte and anolyte with air is excluded, the time of the stable state of $\mathrm{pH}$ and $\mathrm{Eh}$ increases significantly (up to 1 day).

One of the key points of the research was the determination of the displacement of the electrode potential of steel 20 in a corrosive environment with the addition of catholyte, since this makes it possible to evaluate the dynamics of the corrosion process. We used the method of recording polarization curves using an Elins "P-30 S" potentiostat. The stationary electrode potential of steel 20 was measured, which was established in the model of formation water, which was minus $486 \mathrm{mV}$ according to the CAE.

Then, similar tests of steel samples were carried out in the formation water model with the addition of $20 \%$ catholyte. The stationary potential of the sample surface in the model of formation water with catholyte was minus $550 \mathrm{mV}$ according to the CAE. Based on the data obtained, polarization curves were plotted for steel 20 in the formation water model with and without the addition of $20 \%$ catholyte (Fig. 2).

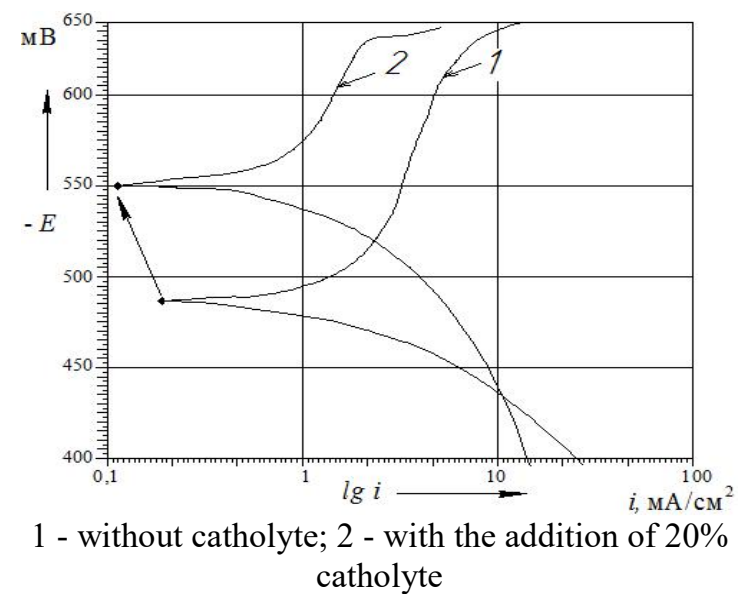

Fig.2. Polarization curves for steel 20 in the formation water model. 
It was found that the addition of $20 \%$ catholyte to the formation water model leads to a decrease in the electrode potential of steel 20 to equilibrium values, as a result of which the corrosion rate decreases due to cathodic polarization. It was revealed that the corrosion rate of steel 20 in the model of formation water with the addition of $20 \%$ catholyte is $0.08 \mathrm{~mm} /$ year, which is 6.7 times less than the corrosion rate in an environment without catholyte. The efficiency of using catholyte as a corrosion inhibitor at a concentration of $20 \%$ is about 85 $\%$. The studies carried out made it possible to obtain the parameters necessary for the development of a unit based on the proposed method for modifying process fluids, which makes it possible to significantly reduce the corrosion rate of oilfield equipment.

Reducing the possibility of salt deposition on the inner surface of oilfield equipment in process fluids can be associated with the process of $\mathrm{pH}$ hydrate formation [8-9]. The addition of catholyte salts to the solutions contributes to a change in $\mathrm{pH}$ to values at which the precipitation of poorly soluble metal hydroxides begins. It was found that when catholyte is added to the model of formation water at a concentration of $30 \%$, a decrease in the content of $\mathrm{Ca}^{2+}$ ions are observed by an average of $69.9 \%$. This is due to a change in the solubility product of the salt cation hydroxide. So, due to the alkalization of the process fluid, hydroxyl ions react more easily with $\mathrm{Ca}^{2+}$ ions, leading to the formation of water-soluble bases with a strong ionic bond.

It was found that to improve demulsification, it is necessary to neutralize the charges of colloidal particles located on the surface of oil globules [10]. The efficiency of separation of emulsions by introducing chemical reagents and anolyte $(10 \%)$ was compared. The use of anolyte (more than $10 \%$ ) as a demulsifier has a high efficiency $-81 \%$. Thus, the addition of anolyte to the oil-water emulsion significantly reduces the time of its separation and does not require the use of chemical reagents and surfactants.

The vital activity of microorganisms largely depends on the electrochemical parameters of the environment in which they live. Thus, many bacteria prefer neutral or alkaline media with $\mathrm{pH}$ values ranging from 6.5 to 9.5 [11]. Therefore, lowering the $\mathrm{pH}$ of the process fluid to 6.0 and below by adding anolyte will help to suppress the vital activity of microorganisms. To determine the viability of cells of sulfate-reducing bacteria (SRB), a process fluid was prepared, contaminated with a pure culture of bacteria Desulfovibrio desulfuricans, simulating formation water. Studies have shown that in a process fluid with a high Eh value (over $250 \mathrm{mV}$ ), SRBs died or fell into anabiotic state. It was found that when at least $20 \%$ of anolyte is added to the contaminated process fluid, the minimum permissible concentration of anaerobic bacteria (100 cells / $\mathrm{ml})$ is reached, and the number of viable SRB cells is reduced to 0 cells $/ \mathrm{ml}$. When its anolyte concentration is $50 \%$, the viability of anaerobic bacteria (including SRB) is completely suppressed.

\section{Conclusions}

The results of the study showed that the corrosion rate of steel in the formation water model with the addition of catholyte at a concentration of $20 \%$ was $0,08 \mathrm{~mm} /$ year, which is 6,7 times less than in a medium without catholyte.

The addition of catholyte to the model of produced water at a concentration of $30 \%$ causes a decrease in the content of $\mathrm{Ca}^{2+}$ ions by about $70 \%$, which is comparable with the use of scale inhibitors.

When anolyte is added at a concentration of $20 \%$ to the process fluid contaminated with microorganisms, the number of anaerobic bacteria decreases by 4 orders of magnitude (up to $10^{3}$ cells $/ \mathrm{ml}$ ), and the number of aerobic microorganisms - on average 8.3 times (no more than $10^{2}$ cells $/ \mathrm{ml}$ ) The addition of anolyte at a concentration of $50 \%$ leads to a complete suppression of the vital activity of microorganisms.

Thus, the use of the developed method and energysaving units for modifying process fluids can prevent or completely eliminate the main complications arising from the operation of oil field pipelines. At the same time, a significant part of the economic effect is achieved by eliminating the use of expensive chemicals and energy-intensive technologies.

\section{References}

1. I. Kostitsyna, A. Shakhmatov, A. Davydov, E3S Web Conf., 121, 04006 (2019)

2. I. Golubev, A. Laptev, E. Alekseeva, N. Shaposhnikov, A. Povyshev, M. Kurakin E3S Web Conf., 121, 02006 (2019)

3. O. Latypov, D. Bugai, E. Boev, Chem. and Petrol. Eng. Vol.51, Iss.3, 283-285 (2015)

4. O. Latypov, f Oilfield Equipment by Management of Electrochemical Parameters of the Medium Chem. and Petrol. Eng. Vol.51, Iss.7. 522-525 (2015)

5. D. Latypova, O. Latypov, D. Bugai, Nanotechnologies in Construction. Vol.10, no.3. 167-178 (2018)

6. N. Skuridin, D. Latypova, M. Pechenkina, O. Latypov, D. Bugai, V. Ryabukhina, Oil industry. 5, 84-86 (2018)

7. A. Tyusenkov, O. Nasibullina, IOP Conf. Ser.: Mater. Sci. Eng. 687, 066016 (2019)

8. M. Pechenkina, D. Latypova, I. Ibragimov, O. Latypov, Oil and gas business, T. 18, № 2, 112-117 (2020)

9. M. Pechenkina, D. Latypova, O. Latypov, D. Bugai, Oil and gas business, T. 18, № 3, 89-96 (2020)

10. O. Latypov, D. Latypova, D. Bugai, V. Ryabukhina, Oil and gas business, T. 14, № 3, 66-71 (2016)

11. O. Latypov, D. Bugai, V. Ryabukhina, Problems of collection, preparation and transportation of oil and oil products, № 2 (104). 28-41 (2016) 